Original article

\title{
The exploration of challenges in clinical knowledge management in nurses: a qualitative study
}

\author{
Kamal Salehi $^{1}$, Sima Kermanshahi ${ }^{2 *}$, Eesa Mohammadi ${ }^{1}$, Mohammad Hassanzadeh ${ }^{3}$
}

(Received: 4 Jan 2015; Accepted: 25 May 2015)

\begin{abstract}
Background and Purpose: Clinical knowledge management (CKM) is considered as a dominant approach for information management and expansion of knowledge in clinical settings. Health care executives have recently begun to focus on CKM. Therefore, identification of challenges against proper CKM planning is of paramount importance. The aim of this study was to explore challenges in clinical knowledge management in nurses.

Methods: This qualitative study was conducted at four teaching hospitals in Tehran, Saqqez and Mahabad, Iran through 24 semi-structured interviews with 18 nurses, head nurses and nursing supervisors. Data analysis was performed using conventional content analysis.

Results: In this study, three main themes emerged from data analysis. The main theme was "organizational culture of less attention to the creation and dissemination of knowledge in nurses", which was due to poor scientific interactions between clinical and academic settings. Other factors involved in this theme were disregard of research in job description of nurses, authoritarian management in research centers, inadequate support and reward from medical officials, and non-observance of research in the evaluation of nurses. Another theme was "poor scientific documentation in nursing", which was due to lack of awareness on the significance of clinical ideas, and proper dissemination of nursing knowledge. Finally, the main theme of "hardness of working conditions" represented high work pressure and lack of opportunity for research among nurses.

Conclusion: It is suggested that nursing managers consider the improvement of organizational culture in strategic plans proposed by the Ministry of Health, in addition to reducing work pressure for nurses. Furthermore, scientific promotion of nursing practice should be taken into account through implementation and documentation of useful and relevant workshops.
\end{abstract}

Keywords: Challenge, Clinical Knowledge Management, Clinical setting, Nurse, Qualitative Study

\section{Introduction}

Some scholars believe the current century to be the era of knowledge management in different fields of human science (1). Knowledge management is a modern concept, which aims at the maintenance, guidance and augmentation of knowledge capitals in various organizations (2). Today, knowledge management has become one of the main concerns of researchers at different associations (3). This is mainly due to the fact that knowledge management leads to creativity and innovation, facilitation of knowledge transfer and preservation of the knowledge of employees before their possible abandonment of the organization (4).

Health care executives have recently focused on knowledge management in different fields of medicine (5). Nurses are one of the major sources of organizational knowledge and essential elements of knowledge transfer in hospitals, operating

\footnotetext{
${ }^{1}$ Department of Nursing, Faculty of Medical Sciences, Tarbiat Modares University, Tehran, Iran

2,* Corresponding author: Department of Nursing, Faculty of Medical Sciences, Tarbiat Modares University, Tehran, Iran. Email: kerman_s@ modares.ac.ir

${ }^{3}$ Department of Management \& Economics, Faculty of Management \& Economics, Tarbiat Modares University, Tehran, Iran
} 
in constantly changing clinical environments. Therefore, enhancement of knowledge plays a pivotal role in achieving professional development, job satisfaction and continuous improvement of nursing practice (6).

Given the critical role of nurses in the acquisition and dissemination of knowledge in health care, clinical knowledge management in nursing practice has gained popularity within recent years (7). The term clinical knowledge management (CKM) was first introduced and discussed by Wyatt in 2001. In a broad sense, CKM is considered as a dominant approach for information management and expansion of knowledge in clinical settings (8); however, accessible plans and measures in this regard are not adequate (9).

In order to be marked as a profession, accumulation of comprehensive knowledge and required skills are of paramount importance in nursing. In a report, Jeremy noted that knowledge management could result in effective decision-making based on the available evidence and innovations in health care systems ultimately increasing the quality of health care measures. Moreover, he postulates that given the remarkable enhancement of medical sciences within the past 20 years, there is a growing need for the implementation of CKM (10).

Application of CKM in clinical environments has numerous benefits, including short length of hospital stay, reduced treatment costs, increased exchange of knowledge between different health care groups and decreased medical errors and. In addition, CKM could help health care professionals to acquire high volumes of information, become aware of recent medical advancements and enhance the quality of care (7). Professional nurses need to be able to create and disseminate new ideas at clinical settings (11). In a qualitative study, Adib Hajbagheri et al. stated the promotion of knowledge and skills as significant factors associated with the empowerment of nursing staff (12).

Despite the significance of knowledge management, few studies have evaluated the challenges against the implementation of CKM. In a review study, Hashemi et al. (2011) concluded that for today's organizations, the most significant challenges associated with knowledge management are finding a precise definition for knowledge, recognition of implicit knowledge and its applications, compatibility with cultural complexities of different populations, consideration of available human resources, planning new organizational structures and coping with increasing competition at work environments (13). In one study, Cong (2003) reported organizational culture, including the values and behaviors of individuals, as a major challenge against the improvement of knowledge management, stating that success of knowledge management depends on the motivation, desire and ability of individuals in sharing and transferring knowledge and information (14).

In another study, Salvati (2009) identified 56 factors affecting knowledge management in state organizations and classified them into three categories of organizational, environmental and citizen factors (15). Due to major differences between health care systems and other organizations, knowledge management could be evaluated more effectively in health care systems in order to obtain more accurate information; however, few studies have been conducted in this regard. For instance, Zárraga believes that every organization is identified with a unique organizational culture, and knowledge management needs to comply with this culture in order to be applicable (16).

In his research, Bali considers the "conveyance of raw data into valuable field information" as one of the most important obstacles against the implementation of CKM (17). In another study, Ghosh (2008) concluded that in comparison to organizational structure, organizational culture has a more significant effect on the function of health care systems (18). In a study performed at public teaching hospitals affiliated to Tehran University of Medical Sciences, Jafari et al. (2011) found a significant correlation between cultural aspects of organization and knowledge management (2).

In another study performed to identify the key competencies for effective implementation of knowledge management in hospitals, Ehsani et al. (2012) claimed that cultural-structural competency plays a pivotal role in the effective implementation 
of knowledge management (19). For the most part, these findings have been reported by quantitative and review studies. Therefore, they may not be able to propose an accurate description of the current situation of CKM in medical systems. In this respect, Cong (2003) argues that knowledge management is a social and human issue inconsistent with positivist approaches, in which large proportions of knowledge might be ignored (14).

By the same token, we applied a qualitative approach in this study to extract in-depth information to obtain accurate and systematic plans for the implementation of CKM. The aim of this study was to explore challenges in clinical knowledge management in nurses.

\section{Materials and methods}

This qualitative study was conducted using conventional content analysis during 2013-2014, and the main research question addressed the challenges against the implementation of CKM in nursing care. Participants were selected by purposive sampling, including nurses, hospital ward staff, nursing supervisors and matrons engaged at Shariati Hospital and Imam Khomeini Hospital (Tehran), Imam Khomeini Hospital (Saqqez county) and Khomeini Hospital (Mahabad city). The only inclusion criterion was having at least two years of work experience in clinical settings. Simultaneous with data collection, data analysis continued until data saturation.

Data collection was performed through conducting semi-structured interviews addressing the following questions:

1. How do you share your experience and knowledge with your colleagues?

2. What challenges have you faced in developing you personal knowledge in nursing practice?

3. How would you normally react to new ideas and suggestions proposed at work? What are the influential factors in this regard?

In addition to the aforementioned questions, subjects were enquired further in more detail. Interviews continued to evaluate the experience level of nurses and its effect on the answers provided to the main research question. Before interviews, objectives of the study were explained to the participants, and the time and place for interview were determined based on mutual agreement. All interviews were meticulously recorded and analyzed by the researchers.

Data analysis was performed using the conventional qualitative content analysis to investigate certain key words and concepts for the reduction and regulation of collected data. In the conventional approach, predefined categories are avoided, and new categories are directly extracted from data (20).

After each interview, transcripts were reviewed several times to extract primary codes. Afterwards, initial codes overlapping with each other were placed in a subsidiary category of sub-themes. Data extraction and deduction of main themes of the study were carried out through further evaluation and comparison of sub-themes based on the statements of participants.

\section{Rigor}

In order to maximize the credibility of our findings, we used prolonged engagement with data (one year), member check, constant comparative and external analyses of data. For dependency, various data extracted from interviews were combined, and for conformability, interview transcripts were evaluated by a panel of experts and multiple observers. Moreover, transferability of data was promoted using maximum variation sample. Study process was recorded step by step in order to obtain conformability, validity, transferability and audit so that the study content could be further explored by other researchers.

\section{Ethical Considerations}

After the approval of study proposal by the Ethics Committee of Tarbiat Modares University, permission was obtained to initiate research. Prior to participation, study objectives and interview methods were explained to the subjects. In addition, participants were assured of confidentiality terms and were allowed to refuse or leave the study at any time. Written informed consent was obtained from 
all the participants for interviews and recordings.

\section{Results}

This study was conducted on a sample size consisting of 11 nurses, 4 head nurses, 2 training supervisors, one matron and one member of ward staff. Subjects were within the age range of 24-48 years (mean age: 35 years), with work experience of 2-26 years (mean: 13 years). In terms of education status, one participant was a $\mathrm{PhD}$ candidate of nursing, two subjects had master's degree, and others had bachelor's degree in nursing. In this study, sampling was performed at different wards of the hospital.

In total, data analysis led to the formation of 182 primary codes, 25 categories, 9 sub-themes and three main themes, which are presented in Table 1. Evaluation of the main themes is as follows:

\section{Organizational Culture of Less Attention to the Creation and Dissemination of Knowledge among Nurses}

This organizational culture is affected by different factors, such as poor scientific interactions between clinical and academic settings, authoritarian management at research centers, disregard of research in job description of nurses, lack of adequate support and reward from medical officials and negligence of research in the evaluation of nurses.

Scientific relations between clinical and academic settings are a significant factor influencing this theme. As such, participants confirmed this parameter as a major challenge associated with the improvement of CKM. It is also noteworthy that this was reported to be a mutual affair; in other words, neither the clinical centers nor the university of nursing had appropriate interactions with each other.

One of the nurses participating in this study said: "Which nurse or nursing authority do you think is related to the university or department of education? The answer is none. If you pay close attention to the way the university envoys students to our hospital, you will notice that we have absolutely no scientific interactions with each other. University officials never offer us a plan to run, or even enquire about our problems at work, neither do hospital authorities."

Another factor affecting organizational culture was reported to be lack of attention to the creation and dissemination of knowledge and disregard of research work in the job description of nurses. According to our participants, research is not considered as an essential part of their job; in other words, conducting research in the field of nursing care would earn these health professionals no specific benefits. In this regard, one of the nurses stated: "So far, I have not been told by anyone that research is part of my profession, or it is my duty to transfer my knowledge of nursing to others. So, I have never done either of the two." In reviewing documents, we also noticed that research work was not proposed as a medical strategy in hospital policy plans.

Presence of authoritarian management at research centers was another factor affecting the appropriate dissemination of knowledge among nurses. According to our participants, apart from research institutes with high-profile physicians in charge, there are no research centers organized for nursing

Table 1. Summary of main themes and sub-themes of clinical knowledge management

\begin{tabular}{ll}
\hline Main Themes & Sub-themes \\
\hline $\begin{array}{l}\text { Organizational Culture of Less Attention to the Creation and Dissemination of } \\
\text { Knowledge in Nurses }\end{array}$ & $\begin{array}{l}\text { 1. Poor Scientific Correlations between Clinical and Academic Settings } \\
\text { 2. Authoritarian Management at Research Centers } \\
\text { 3. Disregard of Research in Job Description of Nurses } \\
\text { 4. Inadequate Support and Reward from Medical Authorities } \\
\text { 5. Negligence of Research in the Evaluation of Nurses }\end{array}$ \\
\hline \multirow{2}{*}{ Poor Scientific Documentationof Nursing Knowledge } & $\begin{array}{l}\text { 1. Inadequate Awareness of Nurses on the Significance of Clinical Ideas } \\
\text { 2. Inadequate Awareness of Nurses on the Registration and Dissemination of } \\
\text { Nardness of Working Conditions }\end{array}$ \\
& 1. High Work Pressure \\
\hline
\end{tabular}


staff to do experiments with the same academic status. Concurrently, this was confirmed as a major obstacle against the transfer of knowledge and information among nursing staff. Nurses mentioned this factor as a deterrent. In this regard, one of the supervisors claimed: "Although several effective medical plans are proposed by nurses on a regular basis, since physicians are the only experts commenting on research work, these plans are commonly rejected or neglected."

Another feature involved in organizational culture is inadequate support of nurses by medical authorities, which was described by the majority of our participants. In theoretical sampling, medical authorities were considered as experts with higher ranks than nurses, including ward staff, chief of wards, supervisors, matrons, chief of hospital and ministers of health. Most of our participants believed that they were not adequately supported by related authorities in different aspects of knowledge management, as one of the nurses remarked: "All authorities theoretically assume a specific position for the development of knowledge, creation of new knowledge or participation in different medical congresses. If asked, these individuals claim they are supportive of knowledge management, while in practice, they barely do anything about it."

Low material or spiritual reward and in many cases, a complete lack of reward were among other factors considered as a challenge against the improvement of CKM in nursing practice. One the nurses in this study said: "So far, no medical authorities have ever considered service charge hours or even verbal appreciation for me for presenting my knowledge to newcomers".

On the same note, another nurse commented: "None of the nurses have enough financial support for initiatives in the field of research or dissemination of knowledge. On the other hand, doctors enjoy substantial budget for conducting experiments, and even if this budget is provided for nursing staff, it is quite insufficient." Interestingly, one of the matrons in this study stated:"We are not capable of providing such financial support for nurses as to conduct research projects."

Another factor involved in organizational culture is taking research into consideration in the evaluation of nursing staff. According to the participants in this study, research has little or no impact on their yearly assessments; therefore, these health professionals tend to be reluctant towards conducting research and experimentation in their field of expertise. One of the nurses in this study claimed: "If conducting research is going to have no impact on the improvement of our annual evaluation by authorities, why would we be willing to do so?"

\section{Poor Scientific Documentation in Nursing Practice}

A major challenge for CKM in nursing practice is poor scientific documentation. In this regard, two main problems were reported by most of the nurses in our study: firstly, some of the nurses were not fully aware of the importance of their clinical ideas and had insufficient knowledge about it, while some others had little information about the registration and dissemination of their knowledge.

On the first area, although many nurses were able to present innovative and useful ideas in a clinical setting, they had mostly lost information sources and therefore, the ideas were no longer available. On the other hand, some nurses were able to present their ideas in various aspects of care and despite reaching the implementation phase, they mostly quit the process since they were not aware of the importance of innovation and new ideas, or were discouraged to put their knowledge into practice. In this regard, one of the nurses remarked: "We did not think that new ideas were important enough to be practiced."

On the second area, most of the nurses claimed that they had little information as to how their ideas could be recorded and disseminated through presentations at related seminars and conferences. One of the nurses said: "For one thing, I did not realize the significance of presenting my knowledge; for another thing, there were no environments where I could be encouraged to publish my research, and the whole process was quite unclear to me. For instance, I did not know how to write scientific papers appropriately since research methodology was not included in our curriculum and it was replaced with 
statistics. Unlike before, research methodology is an essential part of nursing textbooks today."

\section{Hardness of Working Conditions}

Without exception, all nurses in this study noted hardness of working conditions as a significant obstacle against the improvement of CKM. Detailed review of clinical experiences among participants indicated that this factor is mainly associated with high work pressure and lack of professional opportunities to disseminate knowledge.

Heavy workload and tremendous work pressure were noted as significant challenges against the enhancement of CKM, described in words such as "crusher" or "destroyer". One of the nursing managers commented: "Nurses are being crushed under such work pressure at the hospital. How would they find the time to express any new ideas?"

On the same note, one of the members of ward staff stated: "We have shortage of staff. Therefore, I have to work overtime and it is exhausting. When I go home after work, I am not concerned about knowledge, but rather about having some leisure time".

In this regard, another nurse pointed out that working pressure and large number of patients will leave nurses with no time to do research and experimentation. In addition, some nurses mentioned that due to financial problems, they must work in two hospitals, which doubles the work pressure.

Lack of time and opportunity to share information was another major obstacle in the viewpoint of our participants. One of the nurses remarked: "Nurses are too busy dealing with patients and handling care services; so, they hardly have any time to share their experiences at work'. In this regard, another nurse commented: "In a semi-public hospital, there are very many patients and insufficient staff members. So, with such heavy workload, nurses cannot joggle between clinical work and academic projects even if they wished to do so."

\section{Discussion}

According to the results of this study, "organizational culture of less attention to the creation and dissemination of knowledge", "poor scientific documentation" and "hardness of working conditions" are the most significant challenges against the improvement of CKM in the field of nursing.

Based on the experiences of our participants, one of the main obstacles against CKM in nursing practice is organizational culture of less attention to the creation and dissemination of knowledge. In their study, Jafari et al. (2011) concluded that there is a significant relationship between cultural dimensions of health organizations and knowledge management (2).

In another study, Ehsani et al. (2012) claimed that cultural and structural competency could affect the implementation of knowledge management in hospitals (19). In his study, Cong (2003) denoted cultural organization, including values and behaviors of individuals, as the major challenge against the reinforcement of knowledge management (14). Furthermore, Gosh (2008) concluded that organizational culture has a more significant role in health care systems compared to organizational structure (18). Comparison of the results of the aforementioned studies indicates that appropriate organizational culture is the main foundation of knowledge management.

Similarly, findings of the present study highlight the significance of organizational culture in the improvement of CKM. Poor organizational culture is a major obstacle against the creation and dissemination of knowledge. Therefore, it seems that in order for nurses to be able to take advantage of CKM, they should have efficient planning for organizational culture.

With respect to the sub-themes of organizational culture, inadequate interactions between clinical and academic settings were described as a major challenge. In the field of research, collaboration between medical centers and universities is of paramount importance for the promotion of knowledge to improve nursing care (21). Several studies have emphasized the benefits of scientific collaboration between academic and clinical environments, including the higher efficacy of 
nursing interventions and use of evidence-based nursing measures, which ultimately lead to the improvement of patient outcomes (22).

Reinforcement of the body of nursing knowledge is an undeniable necessity for establishing and maintaining nursing schools independent of clinical organizations. In this regard, redefining the role of nursing educators and clinical nurses, as well as creating educational and clinical roles proportional to the level of knowledge and skills based on partnership among the directors of both institutions, could result in the effective relationship between clinical and educational institutions in order to achieve common interests (23).

Among other challenges against the enhancement of CKM were inaccurate job description and disregarding research in the annual evaluation of nurses. Research is the fundamental component of nursing profession and is the basis for development in any field of study (24). Avoidance of doing research by nurses may lead to the stagnation of knowledge, as well as lack of creating national and local knowledge in this field of medicine. In the present study, research projects were not specifically included in the policy plans of hospitals, and this is considered as a noticeable setback in the management of health centers.

According to the results of the current study, authoritarian management and inadequate support were reported as noteworthy challenges against the improvement of CKM. Apparently, this problem is due to the lack of research centers dedicated to nursing experimentation. Therefore, it is recommended that research institutions be established for this purpose with sufficient financial support.

Lack of appropriate rewards for nursing staff was another obstacle mentioned by our participants. Reward systems are key elements to every organization since they help increase employee satisfaction and functionality of an organization. In a study evaluating the effects of rewards on the health care services provided by nurses, Mohammadi et al. (2012) reported that lack of adequate rewards could discourage and make medical staff less eager to work (25).

In another study evaluating job satisfaction among different nurses across the country, Monjamad et al. (2004) observed that $77 \%$ of nurses had low job satisfaction in terms of income and job perks (26). Similarly, the results obtained by Golchin indicated that $88 \%$ of nurses were dissatisfied with their income and benefits. Moreover, the study by Ziapour (2013) reported that $75 \%$ of nurses had average satisfaction with their income and job bonuses (27).

According to the results of the aforementioned studies, rewarding system for nurses is in substandard condition in our country, acting as a significant deterrent against the improvement of CKM. In this regard, balance between material and spiritual rewards should be taken into account. According to the results of the present study, verbal appreciation by authorities is in most cases considered as a reward for nurses. This will motivate the nursing staff and promote their sense of satisfaction. However, in some cases, financial rewards were preferred.

One of the most common complaints on behalf of nurses in the current study was the inequality between the income of physicians and nursing staff. In the study by Mohammadi et al. (2012), sense of inequality was the second most important factor leading to the dissatisfaction of nurses with the reward system (25). In another study, Al-Enzi (2009) claimed that nurses were frequently dissatisfied with extrinsic rewards since they perceived a high sense of inequality for receiving lower income and benefits compared to their colleagues (28). It seems that both material and spiritual rewards concern nurses at health care centers; therefore, balance and equality between rewards should be prioritized in medical organizations.

According to the findings of the present study, poor scientific documentation among nurses was another challenge against the reinforcement of CKM. Documentation is a key element in the process of knowledge management, as well as the creation and maintenance of learning organizations, which will result in operational improvement and excellence of organizations (29).

Documentation leads to creativity and innovation and is an essential factor in converting any association into a learning organization (30). In the 
current study, many nurses were unaware of the significance of their clinical ideas. Perhaps, one of the reasons is lack of knowledge and nourishment of new ideas in nursing education programs, as well as the absence of institutionalization of innovation in courses of nursing bachelor degree. In this regard, some of our participants did not know how to write a research paper; therefore, it is recommended that related workshops be held on research methodology in the educational system of nursing.

Documentation of experience and knowledge accumulates valuable bodies of new information about nursing services, particularly in case of health professionals who are on the verge of retirement and leaving the medical system. Thus, appropriate executive programs should be implemented regarding the documentation of knowledge among nurses. Such programs could be remarkably effective in generating nursing care knowledge.

Hardness of working conditions was one of the most significant challenges against the improvement of CKM, which was mentioned by all our participants without exception. In this respect, problems such as the large number of patients, diverse working shifts and lack of time were pointed out as the obstacles against the enhancement of knowledge management. It is also noteworthy that even nursing managers confirmed that high pressure and heavy workload left them with no extra time to explore any aspects of knowledge management at clinical environments.

Nursing is known to be the second most difficult occupation in the world after mining; therefore, working hours of these individuals should be reduced for physical and mental recuperation (26). Furthermore, nurses need to have more time and enough opportunity so as to be able to explore different aspects of CKM at clinical settings.

\section{Conclusion}

In conclusion, the findings of the present study indicated that poor organizational culture, lack of scientific documentation and hardness of working conditions are the most significant challenges for nurses against the reinforcement of CKM.
Therefore, it is recommended that nursing managers consider the improvement of organizational culture in strategic plans proposed by the Ministry of Health and lay the grounds for the reduction of work pressure in nurses. Moreover, useful workshops could contribute to the promotion of knowledge among nurses in terms of scientific documentation, which will result in the improvement of nursing knowledge both locally and internationally.

\section{Conflict of interest}

None declared.

\section{Authors' contributions}

K Salehi, E Mohammadi, and S Kermanshahi contributed to study concept and design; K Salehi collected the data; S Kermanshahi, K Salehi, E Mohammadi, and M Hassanzadeh analysed and interpreted the data. K Salehi drafted the manuscript; S Kermanshahi, K Salehi, E Mohammadi, and M Hassanzadeh performed critical revision of the manuscript for important intellectual content. S Kermanshahi, E Mohammadi, and M Hassanzadeh supervised the study; moreover, S Kermanshahi, K Salehi, E Mohammadi, and M Hassanzadeh prepared the manuscript. All the authors read and approved the final manuscript.

\section{Acknowledgments}

This study was extracted from a $\mathrm{PhD}$ thesis conducted at Tarbiat Modares University of Tehran. Hereby, we extend our gratitude to Tarbiat Modares University for the financial support of this study. We would also like to thank all the participants for assisting us in this research project.

\section{References}

1. Alavi M, Leidner DE. Review: Knowledge management and knowledge management systems: Conceptual foundations and research issues. MIS Q 2001; 25(1):107-36.

2. Jafari M, Gorji A, Salehi M. The relationship between cultural and structural factors of organizations with 
knowledge management strategy in public teaching hospitals affiliated to Tehran university of medical sciences: 2011. J Heal Adm 2011; 14(45):87-94.

3. Berryman R. Knowledge management in virtual organizations: a study of a best practices knowledge transfer model. Diss, England: University of North Texas; 2005.

4. Anand A, Singh MD. Understanding knowledge management: a literature review. Int J Eng Sci Technol 2011; 3(2):926-39.

5. Kothari A, Hovanec N, Hastie R, Sibbald S. Lessons from the business sector for successful knowledge management in health care : a systematic review. BMC Health Serv Res 2011; 11(1):173-84.

6. Skår R. How nurses experience their work as a learning environment. Vocat Learn 2009; 3(1):1-18.

7. Ghosh B, Scott JE. Effective knowledge management systems for a clinical nursing setting. Inf Syst Manag 2006; 24(1):73-84.

8. Wyatt JC. Clinical knowledge and practice in the information age: a handbook for health professionals. London: RSM Press; 2001.

9. Anderson JA, Willson P. Knowledge management: organizing nursing care knowledge. Crit Care Nurs Q 2009; 32(1):1-9.

10. Wyatt JC. Knowledge management and innovation in medicine: how to go beyond practice guidelines ? Adv Clin Knowl Manag 2002; 5:1-2.

11. Yang CW, Fang SC, Lin JL. Professional knowledge creation in the hospital sector: a qualitative study in Taiwan. Int J Health Plann Manage 2010; 25(2):169-91.

12. Adib Haj Bagheri M, Salsali M, Ahmadi F. The concept of professional power in nursing. KAUMS J 2004; 8(1):9-19.

13. Hashemi SS, Mohammadi Moghadam Y, Mohammadi Moghadam E. knowledge management, requisiteness and Challenges. Q Dev Hum Resour Logist 2011; 6(19):101-30.

14. Cong X, Pandya KV. Issues of knowledge management in the public sector. Electron J Knowl Manag 2003; 1(2):25-33.

15. Salavati A, Haghnazar F. The analytical study of content factors affecting the establishment of knowledge management in Iran national oil company`s central units. Product Manag 2009; 3(10):77-104.

16. Zárraga-Oberty C, De Saá-Pérez P. Work teams to favor knowledge management: towards communities of practice. Eur Bus Rev 2006; 18(1):60-76.

17. Bali RK. Clinical knowledge management: opportunities and challenges. Pennsylvania: IGI Global; 2005.
18. Ghosh B, Scott JE. Knowledge management for healthcare organizations : comparing strategies with technical support. Hawaii: International Conference on System Sciences, Proceedings of the 41st Annual; 2008. P. 1-10.

19. Ehsani A, Moshabaki A. Identification of key capabilities for effective implementation of knowledge management in hospitals with structural equation modeling approach. J Heal Adm 2012; 15(49):58-68.

20. Hsieh HF, Shannon SE. Three approaches to qualitative content analysis. Qual Health Res 2005; 15(9):1277-88.

21. Tubbs-Cooley HL, Martsolf DS, Pickler RH, Morrison CF, Wardlaw CE. Development of a regional nursing research partnership for academic and practice collaborations. Nurs Res Pract 2013; 2013:473864.

22. Balakas K, Bryant T, Jamerson P. Collaborative research partnerships in support of nursing excellence. Nurs Clin North Am 2011; 46(1):123-8.

23. Heshmati Nabavi F, Vanaki Z, Mohammadi E. A critical review on communication paradigms beteween academic and clinical service institutions in nursing. Iran J Med Educ 2012; 12(5):317-30.

24. Yates P, Baker D, Barrett L, Christie L, Dewar AM, Middleton R, et al. Cancer nursing research in Queensland, Australia: barriers, priorities, and strategies for progress. Cancer Nurs 2002; 25(3):167-80.

25. Mohammadi A, Vanaki Z, Memarian R. Assessing the ineffective factors in rewards for nurses: a case study. Q J Nurs Manag 2012; 1(3):46-54.

26. Monjamed Z, Ghorbani T, Mostofian F, Oveissipour R, Nakhost Pandi S, Mahmoudi M. A nationwide study of level of job satisfaction of nursing personnel in Iran. Hayat 2004; 10(4):39-48 (Persian).

27. Ziapour A. An investigation of the job satisfaction and related factors in nurses of Kermanshah hospitals. J Paramed Sci Rehabil 2014; 2(2):27-34.

28. Al-Enezi N, Chowdhury RI, Shah MA, Al-Otabi M. Job satisfaction of nurses with multicultural backgrounds: a questionnaire survey in Kuwait. Appl Nurs Res 2009; 22(2):94-100.

29. Anvari RAA, Shahaei B. Knowledge management and learning organization: analyzing the role of knowledge and experience documentation. J Inf Technol Manag 2009; 1(2):3-18.

30. Elahi S, Baharifar A, Salehi A. Designing a structure for the documentation of managers organizational experiences. Modares Hum Sci 2002; 9(3):23-52. 